\title{
In Strangers We Trust? Findings of an Empirical Study of Distributed Teams
}

\author{
Ban Al-Ani and David Redmiles \\ Donald Bren School of Information and Computer Sciences, University of California, Irvine \\ 444 Computer Science Building \\ Irvine, CA 92697-3440 USA \\ $+1(949) 824-2776$ \\ \{balanilredmiles\}@ics.uci.edu
}

\begin{abstract}
Trust has long been a contentious issue in human endeavours. It is not readily given nor gained, more so when strangers are involved. It often becomes an issue during distributed development where individuals are expected to interact with strangers they may not "meet" during the project lifetime. Trust was spontaneously raised by respondents in an empirical study of practices within distributed development and is reported in this paper. A qualitative analysis of study data suggests that trust typically becomes an issue in large teams when developers are to deliver an innovative product. We also found that it is more likely to be an issue the greater the diversity (of culture, language, time zone...etc.) within the team. Finally the data also suggests that developers more readily trust an authoritative team member (e.g. team leader), even if remote. Data suggests these factors can act as positive and negative forces to influence trust within distributed teams. These forces are reported in this paper together with proposed approaches that can promote equilibrium of the net forces.
\end{abstract}

\section{Introduction}

We conducted an empirical study to investigate some of the challenges encountered and practices adopted by developers working in a large cutting-edge Fortune 500 organization. Three principle areas of practice were investigated during the study, namely: leadership, communication and exchange of ideas. In the process of analysing the data in these different areas, the authors found respondents raised the issue of trust fourteen times although they were not explicitly asked questions relating to trust during the study.

The study discovered four primary forces influence trust during data analysis, namely: team size, type of project, team diversity and leader characteristics with time as a common thread that ran through all these forces. It is not a revelation to find that trust is crucial to both successful collaboration and effective leadership within a distributed group. However, it is interesting that trust was not typically considered a concern by respondents reporting their experience in smaller teams. Furthermore, trust was not generally brought up by respondents involved in further developing products the Organization has been developing for some time. Interestingly, the team's diversity also appears to influence the level of trust in a team. Finally, individuals typically seemed to trust their leader by default and seemed more concerned about gaining the leaders trust than vice versa. These factors can act as forces to influence trust and time emerges as a valuable factor that is needed to allow trust to develop. Recognizing the influence of these forces and reacting accordingly can be one means of accelerating the emergence of trust in distributed teams.

The details of research findings are presented in the following sections of this report together with recommendation derived from existing literature. The second section reviews related work and is followed by an outline of the empirical study and its participants. Research findings related to trust is presented in the fourth section of the paper and is followed by a description of trust from study perspective. The paper ends with a discussion of possible threats to study validity and concluding remarks.

\section{Trust in context}

Trust is a complex term, which can take on varying often overlapping meanings. In general, researchers agree that trust can exist between individuals, individuals and teams, teams and individuals and institutes and thus can be either dyadic or collective $[14,2]$. We will refer to individuals and teams as trustors or trustees in our report.

Ruppel and Harrington [28] review of literature led them to conclude that trust is a dynamic phenomenon that takes on different characteristics during its evolution. They found that it typically goes through 
three stages from the early deterrence-based stage to its more mature stage. In the early deterrence-based stage, there is usually no history or reputation of the trustor or trustee. Thus trust arises out of necessity generally because of a credible threat of punishment for failure to cooperate. This stage is typically followed by another where trust is based on the belief that other the trustees' dispositions are well-known enough that its behavior can be predicted. The final stage is typically identification-based, where the trustor and the trustee understands and agrees with the other's values because of a previous relationship.

Definitions of trust are typically categorized as being rational or social as defined by Jarvenpaa et al [14]. They defined the rational trust as a perspective in which individuals engage in less self-protective actions and are more likely to take risks. We extend this definition to include Wilson et al's [34] definition of cognitive trust, which refers to beliefs about others' competence and reliability, which can lead to individuals engaging in less self-protective actions and are more likely to take risks. The social perspective of trust is defined as a social duty [14]. People will act a certain way to support others because they feel it is the right thing to do or because they feel it is their moral duty. We extend this definition to include Wilson et al's [34] affective trust which arises from emotional ties among trustor and trustee and reflects beliefs about reciprocated care and concerns. Thus, affective trust can lead trustors to act in a way they feel is right. We will refer to these similar categories as cognitive trust in our report.

Finally, our review also led us to conclude that trust can also be swift and/or fragile trust. Swift trust typically arises as a result of the team context, namely: finite lifespan and tight schedule, members with diverse skills, limited history of working together and little prospect of working together in the future $[13,19,12]$. On the other hand Bos et al [2] found that fragile trust is a positive trust that is vulnerable to opportunistic defections and subsequent fallout in their experimental study of collaboration.

We find that all these definitions of trust are presented from a positive perspective. However, we suggest that while positive trust is desirable there are instances where negative trust and mistrust also arises. We derive our definition of positive trust from available literature as a belief that the trustee (individual, team and/or organization) will meet the positive expectations of the trustor (individual, team and/or organization). These expectations can be of the other party's cognitive skills, competencies...etc and thus it can be defined as positive cognitive trust.
Positive affective trust refers to benevolence (care, concern), integrity such that the trustee will not take excessive advantage of the trustor even when the opportunity is available. We suggest that negative cognitive trust occurs when a trustor believes the trustee will not meet commitments and does not have the competencies/skills necessary to contribute to the collaboration effectively. Furthermore, we consider negative affective trust to arise when a trustor believes that the trustee will take advantage of a situation for the trustor's own gain or will generally not be honest or behave with integrity. Negative trust can also arise in the context of dyadic and collective relationships, as the term trustor/trustee is used to refer to one or more individuals or even an organization. We also suggest that in such situations, a trustor experiencing negative trust, will be less likely to take risks and will adopt a defensive stance during collaboration to offset negative behavior. Expectations are set low such that they can be met in such collaborations. Negative trust is different from mistrust in that mistrust typically implies the lack of knowledge and experience of trustees' cognitive or affective behaviors and actions. Expectations will typically reflect average capabilities, competencies and skills when mistrust exists. A let'swait-and-see stance may be adopted in such situations. Therefore mistrust can stem from the unknown and can change to positive trust if expectations are met or exceeded. We feel that negative trust stems from previous negative experiences and will require substantive effort to evolve into positive trust.

Cooperation is considered crucial for the survival of organizations and positive trust is at the heart of cooperation [5, 29]. Positive trust is considered important for collaborators to work effectively and share information openly. It can reduce transaction costs, increase confidence and security in the relationship; promote open, substantive and influential information exchange [14]. Researchers have found that without trust transactions must be carefully contracted and monitored to prevent exploitation, workers change the nature of their collaborations to avoid the need for close coordination, avoid collaboration altogether thus limiting productivity and are less likely to adapt more quickly to changing circumstances, leading to added cost [2, 6, 34].

The importance of trust in distributed software engineering teams has been recognized by several researchers $[17,32,10,21,37]$. Their findings reinforced the critical role trust plays in performance and further found that trust needs to be established early during team evolution by meeting team expectations. While trust can be established early in 
collocated teams this is not necessary true with distributed teams [32]. There is also often an assumption of trust and subsequent strategies are developed based on that assumption [16].

This report's primary focus is on trust amongst distributed developers. The conclusion derived from related literature is a general agreement of researchers and practitioners that an increase in positive trust will typically yield positive results during collaboration.

\section{Empirical study outline}

The research was conducted using an interview format as a survey instrument. The Fortune 500 organization where the interviews were conducted is a well-established leader in computer technology and software development.

Interview questions were developed based on the guidelines detailed by Lorelle and Lawley and Sudman et al [18, 31]. The questions consisted of a mix of open-ended and closed-ended questions. Respondents were always invited and actively encouraged to expand upon their choice, such that context and rational was also gathered.

Individuals were invited to participate in the study through email. A document containing interview questions in addition to research background was attached to the same email. Sixteen employees responded to the invitation to participate. Data revealed that respondents' years of experience within the development domain ranged from 3 to 45 years. Respondents had a mean of 19.3 years experience.

The respondents answered all questions within the context of their two most recent projects (referred to herein as project $\mathrm{A}$ and project $\mathrm{B}$ ). They were informed that they should answer all questions within the context of the team members assigned to project $A$ and project B. Team A was chosen such that all team members were collocated- all members were located in the same city or state. Whereas team B was chosen such that one or more team member was remotely located making it a distributed team. Respondents mentioned a total of 26 different sites. The distributed teams were located on 4 different sites on average

Respondents were also requested to provide an abstract description of the two projects they chose to discuss during the interview. Data revealed that a project deliverable could be one of three different product types, namely: Innovative (a pioneer in the market; no other product like it exists in the world), New (newly developed within the Organization; other organizations have developed a product like it) and Existing (improving a product the Organization is known to develop; a new release of a product currently being developed by the Organization). Our encoded project data is presented in Table 1.

Table 1: Type of project deliverable

\begin{tabular}{|l|l|l|}
\hline Product Type & Collocated & Distributed \\
\hline Innovative & 4 & 1 \\
\hline New & 6 & 8 \\
\hline Existing & 6 & 7 \\
\hline
\end{tabular}

\section{Empirical study findings}

An analysis of data revealed that respondents raised the issue of trust fourteen times during our discussion of distributed development. A limited number of respondents raised the issue of trust across both distributed and collocated teams and even less raised this issue in their discussion of their collocated team alone. These findings led the investigation to focus on trust within distributed teams with a brief reference to trust within collocated teams here.

Trust was raised by a respondent during our discussion of leadership style within the collocated team. He described the following situation:

In A [the collocated team], there were a large number in the end. Early on, there was total empowerment of the team leader, so if he made a decision then management would not second guess that. An engineer can make a decision that something can be done e.g. it's [the product] too small to make, then they trusted that. As they [the team] grew larger they were almost at a stalemate on how to make a decision. Until we realized that it was so bad that both sides decided they need to define how things would be decided in a systematic way.

Trust was typically raised when discussing general leadership attributes desired across both types of teams as illustrated in the following statement:

"...the leader comes out as someone who can be trusted and be willing to take the necessary risk to make the progress, make things happen ..."

These comments reveal that trust is typically a concern in teams in general rather than specific to distributed teams alone. However, the small number of references to trust made by respondents discussing their experiences in a collocated team precluded reaching any meaningful conclusions. Consequently, the research findings are presented within the context of distributed teams alone from this point onwards. The sections highlight the factors that linked the respondents who raised the issue of trust, namely: team size, team diversity, project types and leadership characteristics. 


\subsection{Team size}

The questions concerning teams were generally open-ended to provide respondents with an opportunity to describe their teams in their own terms. Respondents typically gave approximate numbers when defining team size and often stated that team size fluctuated. The maximum number of team members stated at any given time was the number utilized in the calculations that are reported in this section. The authors of this paper found no evidence of prior investigations into the relationship between trust and a distributed development's team size thereby suggesting that this relationship has been overlooked thus far.

An analysis of respondents who raised the issue of trust revealed that large team size was a common factor amongst them. The data revealed that the respondents typically discussed trust when within teams that consisted of an average of fifty-two members. Whereas, the average team size for all teams discussed in this study is thirty-two members. The study data implies that the more developers involved in the development process the more difficult it becomes to establish a sense of trust amongst the distributed team members.

There are indications that the problems associated with large teams are also reflected in communities other than the distributed development teams. For example, leaders in the Hutterite communities found that larger groups are less efficient. Consequently, when the average Hutterite community grows it splits off into two smaller and separate Hutterite communities [7].

The cause of the possible reduction of trust in large teams can be traced to the social capacities of the human brain. Dunbar's [4] findings indicate the constraints on the team size could be a result of the limited ability to recognize and interpret visual signals for identifying either individuals or their behavior. Furthermore, Dunbar's study findings also imply that people are generally limited in their capacity to process emotional information (e.g. cues to other's emotional state). His findings suggest that the limitations of team size are an important component in all human social systems. Dunbar's findings have a direct implication to interactions amongst distributed members. One implication is that distributed members (who typically will not have personal visual contact with remote members) are at an even more severe disadvantage in a large team. The disadvantage is compounded when considered together with the limitations on developers' ability to remember who has a relationship with whom and the ability to manipulate such information for remote team members. This is exemplified in the following statement regarding the potential of meeting face-to-face with remote team members:

"...It makes things very hard, with large groups if there are more than 100 or 50 what's the point? They still wouldn't have that kind of intimacy that they're trying to get through the face-to-face meeting."

In summery, an analysis of study data leads to the hypothesis that trust is more challenging to remote developers working in large teams. One recommendation that suggests itself is that managers apply the "Hutterite Principle" when team size exceeds the recommended average. The findings of this study suggest that the recommended average of a distributed team is less than fifty team members. However, further studies should be conducted before this number can be accepted as an effective distributed team size criterion

\subsection{Projects}

A link between trust and product deliverable type (as defined in section 3 of this report) was also suggested by data gathered during this study. The majority of respondents describing innovative products raised the issue of trust. Fewer respondents describing their experience in teams involved in developing new products raised the issues of trust. Finally, only a minority of respondents describing their experience in teams involved in further developing an existing product raised the issue of trust. This led to the hypothesis that trust was more of a concern when developers were working in relatively uncharted waters (innovative or new products). We found no prior investigation of the relationship between innovativeness and trust within distributed software engineering teams making this a significant finding.

One respondent involved in developing an innovative product explains:

“...It got worse over time, it got more and more second-guessing. There was implied respect for people's ideas. Even managers were second guessed. So there were the managers and the experts' clash of opinions and second guessing an idea...In the same way the non-engineers would second guess the engineers."

The issue of trust might arise in teams involved in innovative and new products because there is a greater need to trust others judgement in addition to the possible fear of presenting new ideas. This conclusion concurs with studies that investigate the relationship between trust and innovation in other domain of study, primarily the business domain. For example, Ruppel and Harrington [28] found that "only trust can assure 
people that they will not be overly penalized for new ideas that fail or that they are free to try improvisations leading to competitive innovations in products, markets, methods, and technologies". Their findings, derived from an empirical study of business managers, seem to echo in distributed development teams concerned with innovative and new products within the Organization.

Holton [11] found that trust in distributed organizational communities develops through frequent and meaningful interaction, where individuals learn to feel comfortable and open in sharing their individual insights and concerns, where ideas and assumptions can be challenged without fear or risk of repercussion and where diversity of opinion is valued.

In conclusions, the data analyzed in this study and literature published in other fields imply the hypothesis that there is a relationship between trust and the development of innovative and new products by distributed teams. The findings of this study suggest that greater emphasis should be given to implementing trust building exercises (similar to those proposed by Pyysiäinen [24] in teams involved in developing innovative products. This finding needs to be explored further to investigate the causal link between innovativeness and trust within distributed teams.

\subsection{Diversity}

Data detailing the locality of distributed team members was also gathered during the study. This data was later analysed and teams were consequently categorized based on diversity. They were categorised as consisting of members being distributed across A) different time zones, B) different cultures, C) different language or D) two or more of the previous three categories.

Overall the majority of the study respondents discussed their experience in teams that fell into category D and most raised the issue of trust. This finding led to the hypothesis that trust was more likely to be an issue within a diverse team.

Examples of trust were generally prevalent when differences in culture or perceived differences in culture (similar to that observed by Vogel [33]) existed. In addition to being closely related to the expected job preservation fears (reported by [9]).

This study observed more of a stereotypical characterization of remote team members. For example, one respondent from Continent (C1) felt that some of the members from another Continent (C2.1) were arrogant and did not trust their (C1) technical knowledge or skill: "...the engineers in the Cl have more tendency to talk longer whereas the engineers in C2.1 for cultural reasons were very impatient to leave when it was [end of the working day in their country]. So it was a struggle to find even a coordinator for the meeting. Here they ended up giving control of the meeting to the people in C2.1 which seemed important to them...culturally there are a lot of assumptions that the C2.1 engineers felt they were superior and a level of arrogance. With this comes a level of mistrust with their C1 counterparts there was a lot of explanations [from C2.1 to C1] "you don't need to know this part of the code you wouldn't understand it"."

Hung et al [12] suggest that the lack of trust in such situations is due to the lack of sufficient time to build proper expectations from prior interactions. They found that people in temporary systems tend to use expectations built on categories reflecting roles, cultural cues, or occupation and identity-based stereotypes. This was also reported by Olson and Olson [22], who state that there is room for misattribution when people from different cultures meet because the cues can be confusing. Whereas, Damian et al [3] found that the lack of trust was exacerbated by differences in organizational culture and history of the relationships between the two sites.

The respondent went on to describe clashes not only amongst team members situated in different continent but also developers working within the same continent but neighbouring countries (e.g. if $\mathrm{C} 2$ is the continent then $\mathrm{C} 2.1$ is one country within $\mathrm{C} 2$ and $\mathrm{C} 2.2$ is another country in the same continent). The previous respondent went on to describe the following situation: "...When the C2.2 came in it added complexity because they also had their own cultural thing where the C2.2 don't like the C2.1 and vice versa. It was so funny, the C2.2 dissect everything and say, no, these guys [C2.1] don't know what they are doing and the C2.1 will say boy those ignorant C2.2, we're going to have to do it our way. The C1 people got caught in the middle. Neither wanted to work with the C1!"

Other respondents reported a lack of trust in communication in general and an unwillingness to share information. The lack of trust can have other negative impacts. For example, one respondent stated that:

“...ideas proposed by members in Cl not colocated with the C2.1 team were not taken up in the beginning. After a year they did, after earning their trust... When they came over to the Cl they did a face to face down in [location X] and were able to get a lot accomplished in that face to face meeting. And build a lot of trust and get some communication going so when 
I did talk with them off-line they knew who I was, they had a face with a name, which is really good..."

The statement indicates that team members had to prove their competence before they were trusted and that trust was further enhanced by a face-to-face meeting. Statements made by respondents also imply that time is a mitigating and potentially positive force; given time trust can be earned as demonstrated in the last statement.

This finding also suggests that Olson and Olson's [21] experimental research into distributed collaborative design can be applicable to distributed collaborative development in general. They found that critical stages of collaborative work, such as establishing mutual trust, appear to require some level of face-to-face interaction as described by one respondent describing a level of distrust even amongst members working in the same country:

"The majority were in the same building. Some were located in [another state]. Again being in a different state created a sense of distrust because they hadn't met the person they were dealing with. It's amazing what face-to-face can do. To find out what they do and what he does as a person. Without this knowledge there is automatically this sense of them and us."

Thus, distrust is not limited to situations where members were in different countries and continents. Respondents also mentioned disparities in culture encountered when interacting with team members in the same country but different states. For example, in a conversation with one respondent compared himself with team members from state $\mathrm{X}$ who appear to be "so different that they might as well be from a different planet". Despite these perceived differences, the likelihood of distrust dissipating is greater in teams that are distributed but remain in close geographic proximity because a greater potential for a face-to- face meeting does exists.

Stewart et al [30] study of teams within business organizations have found that homogeneity is beneficial for teams working on routine tasks but is less beneficial when a team is working on a task requiring a creative solution. Thus, it appears that diversity, which generally stimulates innovation and creativity, is also one of the primary obstacles to effective distributed collaboration as indicated by the findings discussed here. Holton [11], who also conducted a study of teams in business organizations, found that once a team learns to recognize, respect and use its diversity, it can lead to increased opportunities to be innovative, creative and stimulating, if trust can be established within the membership. Holton and Stewart et al found that over time diverse teams can succeed in achieving and even exceeding the performance of homogenous teams.

\subsection{Leadership}

Leadership was defined as being the process through which one member of the group (its leader) influences other group members towards attainment of specific group goals to study respondents [23]. This definition implies that any team member can exercise leadership and influence over their peers, meaning that the existence of a team without leadership is not possible, even though it may not have a formal leader. However, respondents generally readily identified who the team leader was indicating that the person was formally assigned the role and typically demonstrated leadership qualities. Our qualitative data implies that respondents typically trusted their team leader.

Respondents were presented with a series of questions regarding leadership. The questions were a mix of open-ended and closed-ended questions and the respondents were given ample time to elaborate their answers in both situations. For example, respondents were asked to describe the decision making process employed by the leader in both collocated and distributed teams in addition to the impact the locality of the leader had on remote (from the leader) and collocated (with the leader) team members. These questions among others were open-ended. Closedended or scripted questions included the varying types of power the leader exuded to remote and collocated team members and prominent leadership characteristics, among other close-ended questions. The general purpose of these questions was to gain insight on how leaders lead remote team members and whether they require different skill sets from those leading collocated teams. A by-product of these discussions was an impression of how respondents felt about their leader when working in remote localities. One respondent reported the following: “... [The leader] earns the respect and trust of his team members and somebody who can identify the strengths and weaknesses of the team: the capacity to mentor the members. The team is as strong as its weakest contributor. Good people skills: who can identify people's weaknesses and understand them to strengthen their weakness so that the overall team will improve..."

Other similar statements imply that distributed members are more ready to trust a remote leader than a remote peer. This is a significant finding because it means that a leader would have had to work harder for respondents to recall the positive trusting experience 
than the more negative mistrust. Since individuals are more likely to recall and report incidents of trust erosion in comparison to their accounts of trust building incidents; consequently, humans tend to give greater weight to negative entities than to positive entities [27, 15]. In light of this, the generally positive reports gathered from study respondents carry significance weight

The positive influence of the leader has also been reported in previous work. Zhang et al [36] study of virtual teams and leadership style concluded that transformational leadership was associated with higher level of trust in the leader. Their findings also coincide with Raccoon [26] study of developers. They found that developers generally follow those they respect and admire; they demand that leaders warrant their trust.

The contingency theory of leadership that suggests small groups often have two leaders - a task-oriented leader and a socio-emotional leader. Researchers report that in order to predict leadership effectiveness an assessment of situational favorableness also has to be made. Leadership effectiveness depends upon the behavioral style and whether the situation is favorable or unfavorable. Specifically, task-oriented leaders are most effective in highly favorable or unfavorable situations, while socio-emotional leaders are most effective in moderately favorable or more ambiguous situations.

This study's findings regarding leadership and trust is also inline with suggestions proposed in existing leadership literature in other fields of study. Podsakoff et al [24] study of teams in business organizations found that effective leaders can change the basic values, beliefs, and attitudes of followers so that they are willing to perform beyond the minimum levels specified by the Organization. Podsakoff et al findings further suggest that followers who perceive their leaders to provide appropriate models, be supportive, or foster the acceptance of group goals, tend to express more trust in their leaders than employees who perceive their leaders otherwise. Yukl [35], who conducted a study in the same field, concurs with these findings. He also found that leaders who earn the trust and respect toward of their team members will more likely result in greater motivation and for them to do more than they are expected to do.

The data gathered in this study regarding leadership and trust together with the support of related literature can lead to the hypothesis that remote team members are more likely to trust an authoritative figure who demonstrates desired leadership qualities. Data implies that developers can trust a leader in manner that, while not as extreme as those described by Milgram [20], is similar to attributing the responsibility of the decision making process to a person of authority described by Blass [1]. However, literature emphasizes the need for leaders to "lead by example". Existing literature suggests that team members generally expect leaders to meet a model of expected behavior and the ability to rise to meet group rather than personal goals. This ability influences the nature of the relationship between team members (followers) and the team leader. This suggests that team members in leadership positions or aspiring leaders need to meet the basic anticipated characteristics of a leader. Raccoon [26] proposes a list of activities that leaders can adopt to increase team members' trust. These activities can be readily implemented by organizations to prepare potential leaders for the key role they will play in distributed teams.

\section{Trust in study perspective}

Study data provides a vision of the big picture. This picture illustrates the role that the team size, the type of project deliverable and the teams' diversity and leadership skills play in the distributed developers' experience during the distributed development process. The data provides us with an opportunity to crossreference the factors that appear to impact trust within teams and on distributed teams in particular.

The data also reveals that more than one factor exists in each of the respondents' description of their distributed teams size, deliverable and diversity. It is interesting to note that several respondents who raised the issue of trust across both collocated and distributed teams also share commonalities, namely that their collocated teams were also involved in developing innovative of new products within the Organization and that their respective team size was relatively large for collocated teams.

The data implies that there are several forces that inhibit trust in distributed teams. There are also several forces that promote trust. Interestingly, the common denominator that threads these forces is time, which was referred to in several sections of this report. Trust seems to be readily bestowed initially (swift trust [13, 19] yet can break down over time and therefore the problem lies in maintaining it. Time is also the main ingredient in attaining trust in diverse teams, given sufficient time such teams generally perform as well or better than homogenous (collocated) teams and will generally be more creative in achieving tasks.

A collective review of factors that emerged from this study leads to an analogy that these factors act as forces in a manner similar to apposing teams in a "tug- 


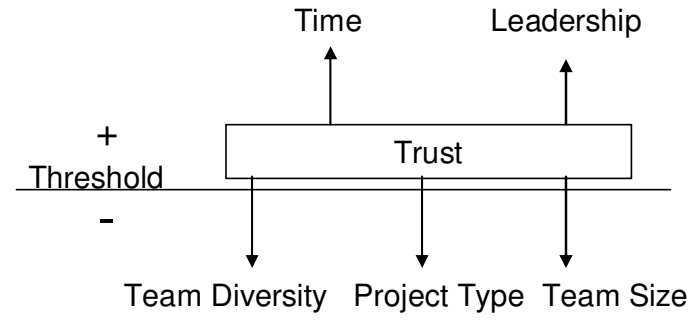

Figure 1: A net force diagram illustrating the positive and negative forces that influence trust.

of-war" with trust placed on the "centre line". Study data presented in this report indicate that a leader who demonstrates anticipated leadership qualities and adequate time allocation can act as two forces that positively influence trust in a distributed team. Conversely, a large team size, high team diversity, and challenging project deliverable can have a negative influence on trust within a distributed team. This tugof-war analogy leads to the conclusion that negative forces can be overcome if the apposing forces (leadership and time) are of sufficient strength and quantity. Study data implies that the key to maintaining equilibrium, at least, is to reach a state such that the trust marking on the rope is closest to positive forces. Trust crossing the centre implies that the trust within the team has negatively crossed over the trust threshold which can imply that the team is not performing to the best of its ability.

Figure 1 is a net force diagram that models the impact of the forces indicated by research findings. The diagram demonstrates that trust is the end result of the net forces acting on it. The trust threshold represents the minimum level of trust necessary for distributed developers to collaborate effectively as a team.

One scenario that figure 1 suggests, is that over an extended period of time a distributed team with high diversity can overcome the potentially negative influence of weak leadership, project type and the drawbacks of large team size. Conversely, if leadership attributes are weak and project development is constrained by time then the project type, team size and diversity may be of greater negative influence within a distributed team. This may lead to trust sinking below the trust threshold which can mean a less effective team performance overall.

Figure 1 currently only includes those forces identified as a result of analyzing research data. However, the figure can be expanded to include other forces (e.g. unequal division of power) identified by other researchers in the field. Future work includes a more in-depth analysis of existing literature to extract forces identified in related studies.

\section{Threats to study validity}

We strove to limit the variables that pose a threat to this study's validity. While potential threats were limited they could not be completely eradicated- three potential threats remained. The first of these threats was the statistical significance of data, which is a subset of the original data set because trust was not a primary research question. While the qualitative data set which led to the research findings is not large, the fact that it came to the surface arbitrarily when analyzing collected data makes it even more significant. A second threat to validity is that the study was conducted in a single organization. However, its size does create a multi-organization diversity. The third limitation is that the research was conducted using an interview format and a survey instrument, which means that collected data relied completely on respondents' ability to accurately recall situations and contexts. Respondents were requested to discuss their most recent projects to limit the impact of this threat.

The limitations of the study can confound the applicability of study findings to a wider population. However, these limitations do not preclude making several observations which can form the foundation of further explorations within this domain.

\section{Concluding remarks}

Humans are trusting when they first become aware of people around them. However, they are generally taught to mistrust everyone around them as they mature. People are generally taught never to give out information over the phone and not to open the door if the person is a stranger. Conversely, trust typically grows exponentially with shared experience, shared friends, and interactions (among others) over a period of time. Yet, humans are usually expected to instantly trust others in the work place. This evolving understanding of trust generally holds true in every culture and community. A great deal of effort must usually be spent to overcome mistrust; more so when individuals are expected to trust people they have never met and seem alien to everything that is familiar to them, as is sometimes the case in distributed teams.

The manner in which the issue of trust was raised in this study indicates that it is still an area of concern within development teams in general and distributed teams in particular (because the higher references to trust within distributed teams). Our study data suggests four hypotheses regarding trust, namely: 
Hypothesis 1. Trust is more likely to be an issue of concern to developers working in large distributed teams.

Hypothesis 2. Trust is more likely to be an issue when developers in a distributed team are to deliver an innovative or new product.

Hypothesis 3. Trust is more likely to be an issue, the greater the diversity of the team's distribution.

Hypothesis 4. Trust is more readily granted to an authoritative team member characterized by leadership qualities within a distributed team.

The hypotheses are presented as separate bullet points but can be closely related. For example, large teams are likely to be distributed in diverse locations and while such a team might be more creative they will also be less trusting of remote members. The hypotheses also suggest that large distributed teams should not be assigned innovative development tasks especially in the initial stages of development and that an attempt should be made to assign a high caliber leader to diverse teams.

These findings can also have significant implication to tools designed to support distributed development. Designers can implement the solutions included in each section that were derived from literature, amongst others, to support the growth of trust within both distributed and collocated teams.

Future work includes conducting research which focuses primarily on investigating trust to test the grounded hypotheses discussed in this report in addition to investigating the existence of other influential forces. Several parallels have already been found between trust within distributed development teams and teams in general, in addition to distributed business teams; these were reported in this paper. Future work should also include extracting and identifying which of these forces are applicable to distributed development. Further research also needs to consider assigning weights to these forces such that the weights reflect their positive or negative strength. Ideally, such research would involve a wider population and be conducted across several organizations. The findings of proposed future work can have significant implications on the kind of support developed and provided for distributed developers.

\section{Acknowledgements}

The empirical study was supported by a P.E.P grant awarded by the University of Technology, Sydney and by the U.S. National Science Foundation under grants 0534775 and 0808783.

\section{References}

[1] Blass, T. Attribution of responsibility and trust in the Milgram obedience experiment, Journal Applied Social Psychology, 26, 1996, 1529-1535

[2] Bos, N., Olson, J., Gergle, D., Olson, G., and Wright, Z. 2002. Effects of four computer-mediated communications channels on trust development. In Proceedings of the SIGCHI Conference on Human Factors in Computing Systems: Changing Our World, Changing Ourselves (Minneapolis, Minnesota, USA, April 20 - 25, 2002). CHI '02. ACM, New York, NY, 135-140.

[3] Damian, D., Izquierdo, L., Singer, J. and Kwan, I., Awareness in the wild: why communication breakdowns occur, Proc. of IEEE Int. Conf. on Global Software Engineering, Germany, Aug 2007, 81-90.

[4] Dunbar, R.I.M The social brain hypothesis. Evolution. Anthropology. 1998, 6: 178-190.

[5] Ferrin, D. L., Bligh, M. C., Kohles, J. C., Can I Trust You to Trust Me? A Theory of Trust, Monitoring, and Cooperation in Interpersonal and Intergroup Relationships, Group \& Organization Management, Vol. 32, No. 4, 465-499 (2007).

[6] Furst, S., Blackburn, R., \& Rosen, B. (1999). Virtual team effectiveness: A proposed research agenda. Information Systems Journal, 9, 249-269.

[7] Gilman, R., Gilman, D., The Farm, Twenty Years Later, Interview with Albert Bates, http://www.context.org/ICLIB/IC29/Bates.htm, accessed July, 2, 2007, Summer 1991, 36.

[8] Grinter, R., Herbsleb, J., \& Perry, D. The geography of coordination: Dealing with Distance in R\&D Work. ACM Conf. on Supporting Group Work, Phoenix, AZ, Nov. 14-17, 1999, 306-315.

[9] Herbsleb J, Grinter R. Architectures, coordination, and distance: Conway's law and beyond. IEEE Software 16(5): 1999, 963-70.

[10] Herbsleb, J., Paulish, D., Bass, M. Global software development at Siemens: experience from nine projects. 27th International Conference on Software Engineering. (St. Louis, MO, USA, May 15 - 21, 2005, 524-533

[11] Holton J.A., Building trust and collaboration in a virtual team, Team Performance Management, Emerald Group Publishing Ltd, 7(3-4), 2001, pp. 3647(12).

[12] Hung, C., Dennis, A., Robert, L. Trust in Virtual Teams: Towards an Integrative Model of Trust Formation. Hawaii international Conference on System Sciences, Track 1, Volume 1 Jan. 5 - 8, 2004.

[13] Jarvenpaa, S. L. and Leidner, D. E. 1999. Communication and Trust in Global Virtual Teams. Organization Science 10, 6 (Jun. 1999), 791-815.

[14] Jarvenpaa, S. L., Knoll, K., and Leidner, D. E. 1998. Is anybody out there? antecedents of trust in global 
virtual teams. J. Manage. Inf. Syst. 14, 4 (Mar. 1998), 29-64.

[15] Lapidot, Y., Kark, R., Shamir, B., The impact of situational vulnerability on the development and erosion of followers trust in their leader, The Leadership Quarterly, 18:1, Feb. 2007, 16-34.

[16] Leszak, M., Meier, M., Successful Global Development of a Large-scale Embedded Telecommunications Product, Proc. of IEEE Int. Conf. on Global Software Engineering, Germany, Aug 2007, to appear.

[17] Lindqvist, E., Lundell, B., Lings, B. "Distributed development in an intra-national, intra-Organizational context: an experience report". In Proc. of the Int. Workshop on GSD for the Practitioner (Shanghai, China, May 23 - 23, 2006). ACM Press, NY, 80-86.

[18] Lorelle, F. and Lawley, M. Questionnaire Design \& Administration. Brisbane: Wiley \& Sons, 2000.

[19] Meyerson, D., K.E. Weick, and R.M. Kramer, Swift trust and temporary groups, in Trust in organizations: Frontiers of theory and research, R.M. Kramer and T.R. Tyler, Editors. 1996, Sage: Thousand Oaks, CA. p. 166-195.

[20] Milgram, S. Behavioral study of obedience. Journal of Abnormal and Social Psychology, 67, 1963, 371-378.

[21] Olson, G., and Olson, J. Distance Matters, In J. M. Carroll (Ed.), HCI in the New Millennium, ACM Press, NY, 2001, 397-417.

[22] Olson, J. and Olson, G., Culture Surprises in Remote Software Development Teams. Queue 1, 9 (Dec. 2003), 52-59.

[23] Pennington, D., The social psychology of behavior in groups, Psychology Press Ltd (2002).

[24] Podsakoff, P. M., MacKenzie, S. B., \& Bommer, W. H. (1996b). Transformational leadership behaviors and substitutes for leadership as determinants of employee satisfaction, commitment, trust, and Organizational citizenship behaviors. Journal of Management, 22(2), 259-298.

[25] Pyysiäinen, J. "Building Trust in Global InterOrganizational Software Development Projects: Problems and Practices", Int. Workshop on GSD, May, 2003, 69-74,

[26] Raccoon, L. B. 2006. A leadership primer for software engineers. SIGSOFT Software Engineering Notes 31, 4, Jul. 2006, 10-15.
[27] Rozin, P., Royzman, E. B. Negativity bias, negativity dominance, and contagion. Personality and Social Psychology Review, 5, 2001, 296-320.

[28] Ruppel C.; Harrington S. "The Relationship of Communication, Ethical Work Climate, and Trust to Commitment and Innovation", Journal of Business Ethics, Springer, 25: 4, Jun 2000, 313-328(16).

[29] Sabherwal, R., "The role of trust in outsourced IS development projects", Communications of the ACM, Vol. 42, No. 2, 1999, pp. 80-86.

[30] Stewart, G., Manz, C., and Sims, H. P. Team Work and Group Dynamics. New York, NY: John Wiley and Sons, 1999, unit

[31] Sudman, S., Bradburn, N. M., Schwarz, N., Thinking About Answers: The Application of Cognitive Processes to Survey Methodology, Wiley \& Sons, Oct. 1995 , chapter 3.

[32] Treinen, J., and Miller-Frost, S., Following the sun: Case studies in global software development, IBM Systems Journal, Vol 45, No 4, 2006, p. 773 - 783.

[33] Vogel, D. van Genuchten, M., Lou, D., Verveen S., van Eekhout, M., Adams, T. Exploratory research on the role of national and professional cultures in a distributed learning project, IEEE Trans. Professional Comm., Jun 2001, 114-125.

[34] Wilson, J.M., Straus, S.G. \& McEvily, W.J. 2006. All in due time: The development of trust in computermediated and face-to-face groups. Organizational Behavior and Human Decision Processes, 99, 16-33.

[35] Yukl, G.A. (1989). Managerial leadership: A review of theory and research. Yearly Review, of Management. $15: 25$ l-289.

[36] Zhang, S., Fjermestad, J. and Tremaine, M., Analysis of Empirical Studies on Leadership Styles in Virtual Team Context: Limitations, Solutions and Propositions, System Sciences, 2005. Proceedings of the 38th Annual Hawaii International Conference, 0306 Jan. 2005, 48c - 48c.

[37] Zheng, J., Veinott, E., Bos, N., Olson, J. S., and Olson, G. M. 2002. Trust without touch: jumpstarting longdistance trust with initial social activities. In Proceedings of the SIGCHI Conference on Human Factors in Computing Systems: Changing Our World, Changing Ourselves (Minneapolis, Minnesota, USA, April 20 - 25, 2002). CHI '02. ACM, New York, NY, 141-146. 